\title{
FDTD modeling to enhance the performance of an organic solar cell embedded with gold nanoparticles
}

\author{
Chung-How Poh, ${ }^{1, *}$ Lorenzo Rosa, ${ }^{2}$ Saulius Juodkazis, ${ }^{2,3}$ and Paul Dastoor ${ }^{1}$ \\ ${ }^{l}$ Centre for Organic Electronics, University of Newcastle, Callaghan, NSW 2308, Australia \\ ${ }^{2}$ Centre for Micro-Photonics, Faculty of Engineering and Industrial Sciences, Swinburne University of Technology, \\ Hawthorn, VIC 3122, Australia \\ ${ }^{3}$ Melbourne Centre for Nanofabrication, 151 Wellington Road, Clayton, VIC 3168, Australia \\ *Chung-How@ieee.org
}

\begin{abstract}
Optical enhancement is demonstrated in a bilayer P3HT- $\mathrm{C}_{60}$ solar cell by embedding gold nanoparticles directly into the P3HT layer of the photovoltaic device. FDTD simulations are used to model the observed performance gain. A qualitative agreement between the experimental and numerical results is achieved. This validates the numerical model and the simulation is subsequently extended to predict the performance gain of the bilayer device constructed with thinner P3HT layer. The numerical results reveal that the plasmonic structure has even larger effect on such thinner bilayer device. The enhancement is expected to be most significant when the $\mathrm{p}-\mathrm{n}$ interface is allowed to assume the conformal hemispherical profile of the metal particles.
\end{abstract}

C2011 Optical Society of America

OCIS codes: (250.5403) Plasmonics; (310.6628) Subwavelength structures, nanostructures; (040.5350) Photovoltaic.

\section{References and links}

1. Y. A. Akimov, W. S. Koh, and K. Ostrikov, "Enhancement of optical absorption in thin-film solar cells through the excitation of higher-order nanoparticle plasmon modes," Opt. Express 17(12), 10195-10205 (2009).

2. V. E. Ferry, M. A. Verschuuren, H. B. Li, E. Verhagen, R. J. Walters, R. E. Schropp, H. A. Atwater, and A Polman, "Light trapping in ultrathin plasmonic solar cells," Opt. Express 18(S2), A237-A245 (2010).

3. X. Li, N. P. Hylton, V. Giannini, K. H. Lee, N. J. Ekins-Daukes, and S. A. Maier, "Bridging electromagnetic and carrier transport calculations for three-dimensional modelling of plasmonic solar cells," Opt. Express 19(S4), A888-A896 (2011).

4. A. J. Morfa, K. L. Rowlen, T. H. Reilly, M. J. Romero, and J. van de Lagemaat, "Plasmon-enhanced solar energy conversion in organic bulk heterojunction photovoltaics," Appl. Phys. Lett. 92(1), 013504 (2008).

5. S.-S. Kim, S.-I. Na, J. Jo, D.-Y. Kim, and Y.-C. Nah, "Plasmon enhanced performance of organic solar cells using electrodeposited Ag nanoparticles," Appl. Phys. Lett. 93(7), 073307 (2008).

6. D. Duche, P. Torchio, L. Escoubas, F. Monestier, J.-J. Simon, F. Flory, and G. Mathian, "Improving light absorption in organic solar cells by plasmonic contribution," Sol. Energy Mater. Sol. Cells 93(8), 1377-1382 (2009).

7. A. J. Morfa, T. H. Reilly, J. C. Johnson, and J. van de Lagemaat, "Plasmons in solar energy conversion," (SPIE Newsroom, 2009), http://spie.org/x35474.xml?ArticleID=x35474.

8. T. H. Reilly, J. van de Lagemaat, R. C. Tenent, A. J. Morfa, and K. L. Rowlen, "Surface-plasmon enhanced transparent electrodes in organic photovoltaics," Appl. Phys. Lett. 92(24), 243304 (2008).

9. M. A. Sefunc, A. K. Okyay, and H. V. Demir, "Plasmonic backcontact grating for P3HT:PCBM organic solar cells enabling strong optical absorption increased in all polarizations," Opt. Express 19(15), 14200-14209 (2011).

10. W. Bai, Q. Gan, G. Song, L. Chen, Z. Kafafi, and F. Bartoli, "Broadband short-range surface plasmon structures for absorption enhancement in organic photovoltaics," Opt. Express 18(S4), A620-A630 (2010).

11. N. N. Lal, B. F. Soares, J. K. Sinha, F. Huang, S. Mahajan, P. N. Bartlett, N. C. Greenham, and J. J. Baumberg, "Enhancing solar cells with localized plasmons in nanovoids," Opt. Express 19(12), 11256-11263 (2011).

12. M. O. Reese, A. J. Morfa, M. S. White, N. Kopidakis, S. E. Shaheen, G. Rumbles, and D. S. Ginley, "Pathways for the degradation of organic photovoltaic P3HT:PCBM based devices," Sol. Energy Mater. Sol. Cells 92(7), 746-752 (2008).

13. K. R. Catchpole and A. Polman, "Plasmonic solar cells," Opt. Express 16(26), 21793-21800 (2008). 
14. P. N. Saeta, B. I. Greene, A. R. Kortan, N. Kopylov, and F. A. Thiel, "Optical studies of single-crystal C 60 ," Chem. Phys. Lett. 190(3-4), 184-186 (1992).

15. K. R. Catchpole and A. Polman, "Design principles for particle plasmon enhanced solar cells," Appl. Phys. Lett. 93(19), 191113 (2008).

\section{Introduction}

Plasmonic structure has been widely used to enhance the performance of silicon-based solar cells [1-3]. Various architectures have also been devised to achieve plasmonic effect in organic solar cells, with silver commonly used as the plasmonic element. One of the demonstrated architectures is by having the plasmonic element in the PEDOT:PSS layer [4,5]. Another possible architecture is to deposit the plasmonic element within the photoactive layer $[6,7]$ but since this layer is usually composed of a random intermixing of $p-n$ junction, the presence of the electrically conducting element would often lead to substantial charge recombination and internal short [7]. Notably, the plasmonic enhancing effect has also been achieved by employing a patterned metallic layer as part of the counter electrode [8-10].

In this paper we propose to implement the plasmonic element within the photoactive layer, based on a bilayer structure as shown in Fig. 1. The metal particles are confined within the ptype semiconductor region to prevent charge recombination and internal short. The strategic location of the metal particles will also permit the local field enhancements of the particles to be harvested. Unlike certain approaches [11], this proposed configuration enables a generous amount of light to get into the charge generating layer of the solar cell. The thickness of the $\mathrm{C}_{60}$ layer can be tuned so that the $\mathrm{p}-\mathrm{n}$ junction is at an appropriate distance from the reflective $\mathrm{Ca} / \mathrm{Al}$ electrode. This ensures maximum light intensity developed across the $\mathrm{p}-\mathrm{n}$ junction. Instead of silver, gold was chosen in this work as the plasmonic element (in the form of gold nanoparticles) given its stability as well as compatibility with the photoactive layer [12,13].

\section{Experimental procedures}

\subsection{Device fabrication}

Two separate substrates were prepared and were identical except one had gold nanoparticles embedded between the PEDOT and P3HT layers, as illustrated in Fig. 1. The bilayer device was built on an indium tin oxide (ITO) coated glass substrate, which was cleaned with cotton tip and isopropanol prior to use. The Baytron ${ }^{\circledR} \mathrm{P}$ PEDOT:PSS [poly(3,4ethylenedioxythiophene) poly(styrenesulfonate)] was spun at $4000 \mathrm{rpm}$ to yield a film thickness of about $30 \mathrm{~nm}$. The spun film was immediately transferred into a glove-box and thermally annealed at $160{ }^{\circ} \mathrm{C}$ for 10 minutes. Thermal evaporation was used to deposit the gold nanoparticles. The evaporator shuttle was opened for $2 \mathrm{~s}$ once the flux had stabilized at $0.5 \AA / s$. As a result nanoparticles were formed on the PEDOT:PSS surface. Once the thermal evaporation had been completed, a $140 \mathrm{~nm} \mathrm{P3HT} \mathrm{[poly(3-hexylthiophene)]} \mathrm{film} \mathrm{was}$ deposited. The sample was subsequently returned to the evaporator for depositing the $\mathrm{C}_{60}$ layer. Finally, the $\mathrm{Ca} / \mathrm{Al}$ electrode was added to complete the photovoltaic device. The completed device is as shown in the inset of Fig. 3 and it consists of four independent cell regions for characterization purpose. The area of each cell measured $3.5 \times 4.0 \mathrm{~mm}^{2}$.

\subsection{Characterizations}

The completed device was tested under Air Mass 1.5 simulated solar illumination. Keithley's SourceMeter 2400, with a LabVIEW interface were used to obtain the $I-V$ curves of each cells. The data were logged to a computer.

The surface morphology of the deposited gold layer was studied using an atomic force microscope (AFM) operating in the tapping mode. The AFM tip was silicon ( $\mathrm{Si}$ ) with a $\mathrm{Si}_{3} \mathrm{~N}_{4}$ coating. The scan area was $5 \times 5 \mu^{2}$, and the line scan resolution was set to 512 . All of the AFM measurements were performed under ambient conditions. 


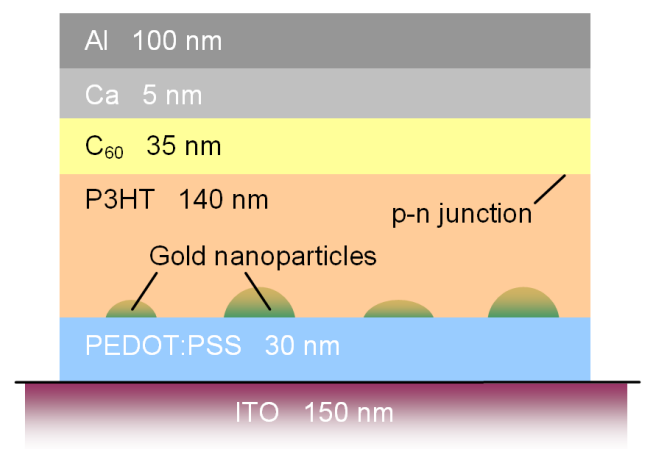

Fig. 1. Geometry of the fabricated organic solar cell.

\section{FDTD simulations}

Numerical simulation of light intensity distribution in the solar cells were carried out using a FDTD solver (Lumerical Solutions, Inc). Spectral dependencies of optical properties of materials, the refractive index and absorption coefficient, were taken from the FDTD solver database or imported from available literature, e.g., $\mathrm{C}_{60}[14]$.

The model was based on the geometry in Fig. 1, in which a linearly polarized light enters the photovoltaic cell through the glass substrate. The simulated wavelength ranged from 340 to $560 \mathrm{~nm}$. The propagating optical power inside the cell structure was measured with three monitors $P_{A}, P_{B}$ and $P_{R}$.

$P_{A}$ was placed $35 \mathrm{~nm}$ above the $\mathrm{P} 3 \mathrm{HT} / \mathrm{C}_{60}$ interface to collect the power not absorbed in the depletion zone, which was considered to extend $35 \mathrm{~nm}$ above and below the interface. $P_{B}$ was placed $50 \mathrm{~nm}$ below the nanoparticle layer to collect any optical power impinging on the nanoparticles. Lastly, $P_{R}$ was used to collect any optical power reflected from the solar cell structure. The absorbance, $A$ within the photoactive layer was evaluated as:

$$
A=\left(1-P_{R}\right) \cdot \frac{P_{B}-P_{A}}{P_{B}}
$$

The modeling was performed for cases involving different particle sizes. The radii modeled in this work were $35,50,75$, and $100 \mathrm{~nm}$. We also included a case whereby the radii of the metal particles in the solar cell were randomly distributed in the range of 30 to $50 \mathrm{~nm}$. The results of the FDTD modeling were presented in terms of the difference in the absorbance percentage between the cases with and without the nanoparticles.

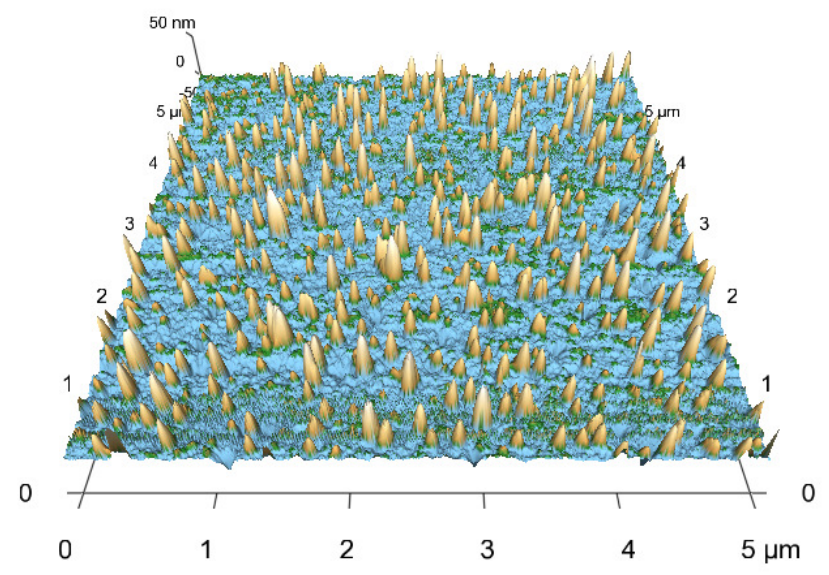

Fig. 2. AFM image of the gold nanoparticles evaporated onto the PEDOT:PSS layer. 


\section{Results}

\subsection{With $140 \mathrm{~nm}$ P3HT layer}

Gold nanoparticles of hemispheric and oblate in shape were observed by AFM on the PEDOT:PSS surface after evaporation (Fig. 2). The diameter of the particles was found to be around 80 to $120 \mathrm{~nm}$ while the typical height ranged from 20 to $40 \mathrm{~nm}$. Such size and shape are expected to be near ideal for light scattering and trapping [15].

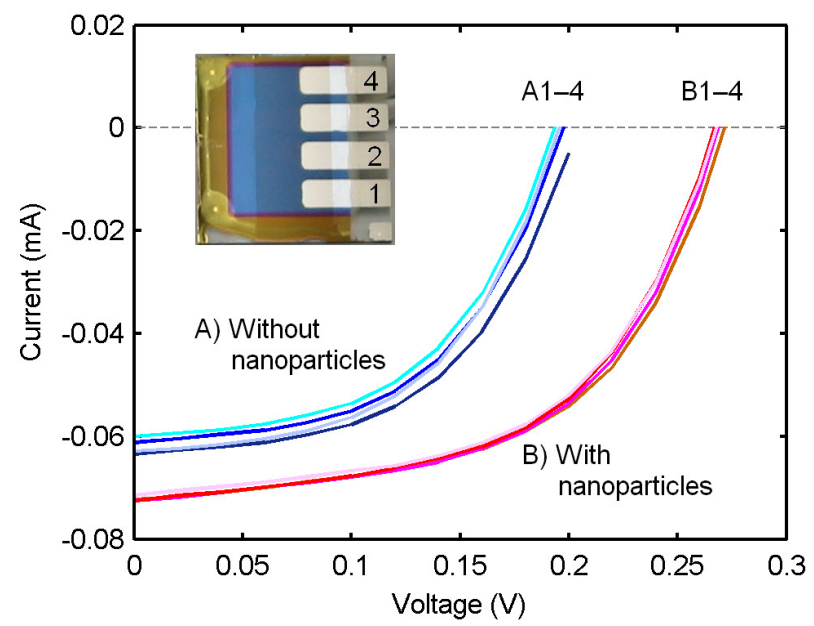

Fig. 3. I-V characteristic of solar cells without and with gold nanoparticles. Thickness of the P3HT layer was $140 \mathrm{~nm}$. Four closely spaced curves represent four separate solar cells with and without nanoparticles, respectively (the cells are shown in the inset).

The effects of the nanoparticles on the $I-V$ curves are presented in Fig. 3. Table 1 shows the performance parameters. Sample B, with nanoparticles demonstrated an increase in all of the device parameters. The $140 \mathrm{~nm}$ P3HT thickness was chosen to ensure that internal shorts are avoided, which otherwise would render the sample useless. However, such P3HT thickness is not optimized from the view point of charge extraction as the diffusion length is limited to about $10 \mathrm{~nm}$. With a thinner P3HT layer, the open circuit voltage, $V_{o c}$ will naturally increase to about $300 \mathrm{mV}$ (without the nanoparticles). It is not yet clear at this stage whether the higher $V_{o c}$ of the thinner bilayer will still be affected by the addition of the gold nanoparticles. It is thought that the open circuit voltages in Table 1 were improved in the presence of the nanoparticles by the formation of a Schottky barrier.

Table 1. Performance of the Solar Cells: A) without and B) with Gold Nanoparticles

\begin{tabular}{ccccc}
\hline Cell & PCE (\%) & FF & $J_{s c}\left(\mathrm{~mA} \cdot \mathrm{cm}^{-2}\right)$ & $V_{o c}(\mathrm{~V})$ \\
\hline A-1 & 0.057 & 0.528 & 0.528 & 0.203 \\
A-2 & 0.053 & 0.522 & 0.510 & 0.198 \\
A-3 & 0.050 & 0.515 & 0.500 & 0.194 \\
A-4 & 0.053 & 0.520 & 0.524 & 0.196 \\
B-1 & 0.090 & 0.551 & 0.602 & 0.272 \\
B-2 & 0.089 & 0.550 & 0.604 & 0.269 \\
B-3 & 0.088 & 0.544 & 0.605 & 0.267 \\
B-4 & 0.087 & 0.543 & 0.596 & 0.268 \\
\hline
\end{tabular}

PCE: Power conversion efficiency, FF: Fill factor

$\boldsymbol{J}_{s c}$ : Short circuit current density, $\boldsymbol{V}_{o c}$ : Open circuit voltage

(C) 2011 OSA
Received 1 Sep 2011; revised 7 Oct 2011; accepted 13 Oct 2011; published 21 Oct 2011 1 November 2011 / Vol. 1, No. 7 / OPTICAL MATERIALS EXPRESS 1329 


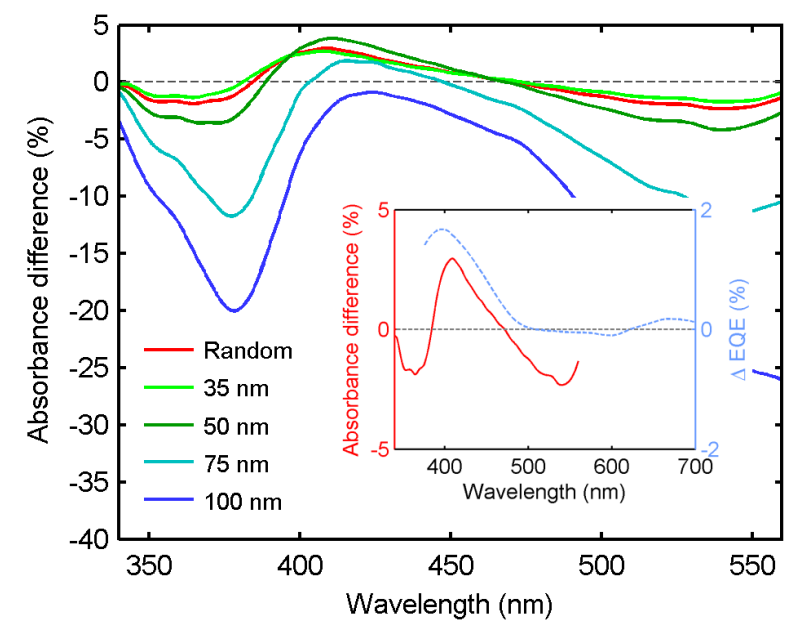

Fig. 4. Absorbance difference corrected for reflection with respect to the case without nanoparticles. The inset compares the experimental EQE against the FDTD result.

The inset of Fig. 4 (blue dotted curve) shows the difference in the external quantum efficiency, $\triangle \mathrm{EQE}$ between the cases with and without the nanoparticles. There are two positive peaks in the measured $\triangle \mathrm{EQE}$, centered around 395 and $675 \mathrm{~nm}$. These wavelengths correspond respectively to 1) the absorption of gold via the intra-band transitions, and 2) the plasmon resonance of the nanoparticles. However, it can be seen that the size of the resonance peak around $675 \mathrm{~nm}$ is relatively insignificant when compared to that of the absorption peak at $395 \mathrm{~nm}(\triangle \mathrm{EQE}=0.18$ vs. $1.80 \%)$. This is because the rather large distance $(\sim 100 \mathrm{~nm})$ between the nanoparticles and the p-n junction (as shown schematically in Fig. 1) has minimized the influence of the local field enhancement of the nanoparticles. Therefore, it was determined that the improved $I-V$ characteristics as observed in Fig. 3 were mainly due to the light absorption of the gold nanoparticles.

In our previous study, adding a continuous layer of gold (i.e. without the nanoparticles) into the solar cell produced no observable increase in the EQE. This ascertains the fact that the increase in the EQE is attributed to the presence of the nanoparticles. Furthermore, this also confirms that the gold layer is compatible with the structure, probably because of the high electronegativity of gold and its proximity to the anode (the ITO).

Since the field enhancement effect is comparatively minor due to the $140 \mathrm{~nm}$ P3HT layer, the FDTD simulation has primarily focused on the absorption part of the spectrum (350 to 560 $\mathrm{nm}$ ). The numerical results for the various radii are as presented in Fig. 4. Absorbance in the positive direction in the figure denotes improved absorption of incident light by the photoactive layer (the $\mathrm{P} 3 \mathrm{HT}$ and $\mathrm{C}_{60}$ layers). The solar cells were modeled in terms of absorbance because the absorbance is related to the measured EQE. Thus, a higher absorbance implies that more photons are being absorbed in the photoactive layer and therefore giving rise to a larger photocurrent, i.e. an improved $\mathrm{EQE}$ at that particular wavelength.

The simulated "Random" curve in Fig. 4 is of particular interest because it modeled a set of randomly distributed nanoparticle radii from 30 to $50 \mathrm{~nm}$ which closely resembled the thermally evaporated nanoparticle layer. The "Random" curve was singled out and included in the inset of Fig. 4 to compare against the profile of the experimentally obtained EQE. The inset shows that the simulated "Random" curve was qualitatively in good agreement with the measured EQE. The simulated data predicted an improvement in the absorbance around 410 $\mathrm{nm}$ wavelength and this was not far off from the observed experimental value of $395 \mathrm{~nm}$ (blue dotted curve). This lends support to the correctness of the numerical model. Moreover, the experiment revealed that the sample with nanoparticles had a negative $\triangle \mathrm{EQE}$ for wavelengths longer than $510 \mathrm{~nm}$. This trend was also correctly predicted by the FDTD model albeit with a higher loss in EQE. 
FDTD analysis indicated that the local field enhancement is influential up to about $10 \mathrm{~nm}$ from the surface of the nanoparticles. Therefore, a device with a much thinner P3HT layer is required before the local field enhancement will become significant.

\subsection{With thinner $40 \mathrm{~nm}$ P3HT layer}

Given that the FDTD model has yielded satisfactory results, the simulation was extended to model a similar structure but with a thinner $40 \mathrm{~nm}$ P3HT layer. The depletion zone in the computer model was reduced to $35 \mathrm{~nm}$ thick. We considered three possible scenarios which are depicted in Fig. 5: (1) the P3HT and the $\mathrm{C}_{60}$ layers are both flat, (2) only the P3HT layer follows the hemispherical profile of the nanoparticles, and (3) both layers assume the conformal hemispherical profile. The results of the simulation are shown in Fig. 5.

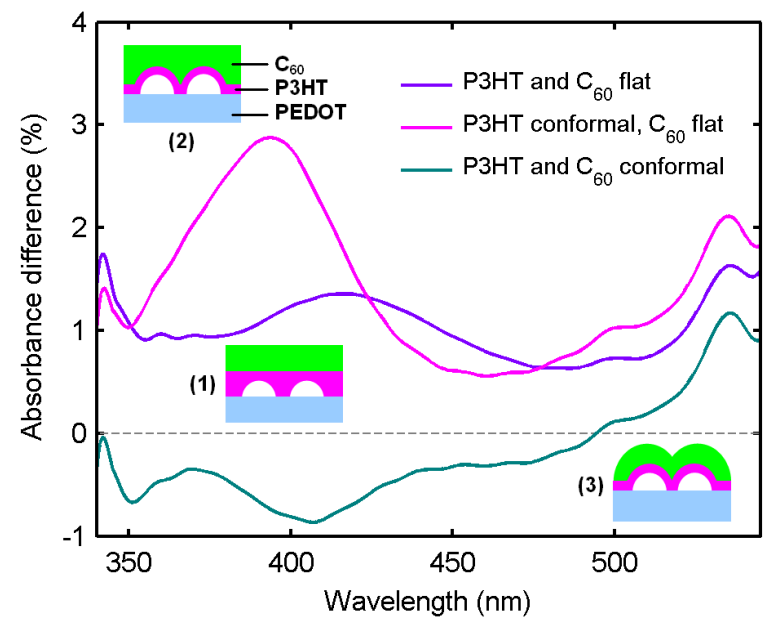

Fig. 5. Absorbance difference corrected for reflection with respect to the case without nanoparticles for thinner $40 \mathrm{~nm}$ P3HT layer and $35 \mathrm{~nm}$ depletion zone.

Out of the three scenarios, scenario (2) gives the best overall performance in terms of expected efficiency enhancement. The simulation predicted that there would be a prominent peak occurring just below $400 \mathrm{~nm}$. FDTD analysis indicated the presence of intense field around the nanoparticles reaching into the vicinity of the $\mathrm{p}$-n junction.

Scenario (3) gives the least performance gain, and in fact its absorbance difference was predicted to be negative for most of the simulated spectral range. As for scenario (1), the gain in the absorbance was only moderate. The gain is expected to be small but fairly uniform across the spectral range.

\section{Conclusion}

The increase in the external quantum efficiency of organic solar cells has been numerically modeled and a satisfactory agreement between the theory and experiment has been achieved. It was established that with the $140 \mathrm{~nm}$ P3HT bilayers the enhanced $I-V$ characteristics were primarily due to the optical absorption of the gold via the intra-band transitions. The FDTD model was subsequently extended to predict the performance of the solar cells constructed with thinner P3HT layer. The FDTD modeling suggested that plasmonic field enhancement would have even stronger effect on the performance of such solar cells. 